\title{
SELF-IMAGE AND SEXUALITY ISSUES AMONG YOUNG WOMEN WITH BREAST CANCER: PRACTICAL RECOMMENDATIONS
}

\author{
Clara Hungr ${ }^{1}$, Veronica Sanchez-Varela and Sharon L. Bober ${ }^{1 *}$ \\ 1Department of Psychosocial Oncology and Palliative Care, Dana-Farber Cancer Institute/Harvard Medical School, \\ Boston, USA
}

\begin{abstract}
Background: Younger breast cancer survivors face a unique set of treatment-related issues that have enormous impact on quality of life and psychological well-being. Although there is often a profound and distressing impact of treatment on self-image and sexual function, women rarely receive any attention for these issues. Unfortunately, most clinicians receive no training on how to inquire about these problems and most clinicians feel unprepared to discuss them. Often this silence is due to a lack of ready resources and uncertainty of appropriate strategies for rehabilitation. Cultural barriers may also contribute to lack of attention to these issues. Objective: The aim of this paper is to not only elucidate common problems regarding self-image and sexual dysfunction, but to also offer concrete guidance about inquiry using a simple checklist approach as well as tips for resources and other evidence-based intervention strategies. Samples of a checklist and resource sheet for women written in Spanish are included, and cultural considerations within a Hispanic/Latina framework will be noted. As the great majority of young breast cancer patients are now becoming long-term survivors, it is essential for clinicians to learn how to address distressing treatment-related late effects including diminished self-image and sexual dysfunction. (REV INVES CLIN. 2017;69:114-22)
\end{abstract}

Key words: Self-image. Body image. Sexual functioning. Breast cancer survivor. Cultural considerations.

\section{INTRODUCTION}

Breast cancer is currently the most commonly diagnosed cancer for young women between the ages of 15 and $39^{1}$. Despite the high incidence, advances in breast cancer screening and treatment have led to prolonged survival, with five-year rates around $90 \%^{2}$. However, the very treatments that allow young women to live cancer-free come with a range of longstanding physical and psychological difficulties. Young survivors face unique challenges to their quality of life in the survivorship phase of care, including body image and sexual function. These experiences must be understood and addressed in order to help young women live full and satisfying lives after breast cancer.

Breast cancer treatment typically involves full or partial surgical resection of the breast, chemotherapy,

\section{Corresponding author:}

*Sharon Bober

Sexual Health Program

Dana-Farber Cancer Institute/Harvard Medical School

Shields-Warren 320, 450 Brookline Ave

Boston, MA, 02215, USA

Email: Sharon_Bober@dfci.harvard.edu

Received for publication: 01-03-2017

Approved for publication: 17-03-2017 
and radiation. Additional hormonal (anti-estrogen) treatment is also given to the majority of women with estrogen receptor (ER)-positive cancers ${ }^{3}$. Due to biological differences in breast tissue, compared to their older cohort, young women generally face more aggressive cancers and subsequently more aggressive courses of treatment ${ }^{4}$. After these treatments, young breast cancer survivors typically contend with various physical changes, including partial or complete loss of the breast, premature menopause, and subsequent changes in sexual functioning and fertility ${ }^{5}$. The need to face changes that can be both dramatic and abrupt, and subsequently make adjustments during a time in life when good health is generally assumed, has far-reaching psychological implications. In particular, we will focus on body image and sexual function, both of which have enormous impact on a woman's overall sense of femininity and self.

It is important to note that women's psychological experience of sexuality and body image is also integrated within a broader socio-cultural context ${ }^{6}$. Along with understanding women's general experience of changed womanhood after cancer, it is vital to understand how this experience plays out within a particular cultural environment. This paper will review the impact of breast cancer on the self-image of young female survivors, with an emphasis on changes to body image and sexual function. The review will include a discussion of how these issues may be experienced specifically within the Hispanic/Latino culture. Finally, this article will provide practical suggestions and resources for clinicians on how to engage young breast cancer survivors around the sensitive issues of self-image and sexuality.

\section{SELF-IMAGE}

Broadly speaking, disruptions to self-image occur when the "social assumptions which define (survivors) as women no longer match their own interior definitions of what it means to be a woman" ${ }^{\text {" }}$. In general, the experience of a familiar sense of the body, the self, and social interactions come together to embody a view of "me." From this "me," an expectation develops of how the body will function and respond in certain situations. When this familiar definition of the self is interrupted by the physical changes of cancer, especially in a younger woman who expects a healthy and functional body, the self-image no longer feels familiar and safe ${ }^{8}$.

The self-image of a young breast cancer survivor is influenced by both physical and psychological components. Both of these elements can be examined independently but with the understanding that they are overlapping constructs. In other words, the experience arising from the physical body informs a woman's subjective sense of femininity and attractiveness, and of being able to enjoy the body as a medium for self-expression and way of being in the world 9 . Moreover, a young woman's subjective perceptions of herself may have an enormous influence on how her physical body responds. Although an artificial distinction, self-image can be examined through the lens of both of the above components, specifically through the psychological construct of body image and the physical aspects of sexual functioning. Below, these are reviewed separately in order to help elucidate a woman's breast cancer experience.

\section{BODY IMAGE}

Broadly speaking, body image is defined as the way in which a woman perceives and evaluates the integrity of her physical body. This encompasses an attitude about the body being functional, whole, and healthy ${ }^{10}$. For young women who undergo breast cancer treatment, significant body changes can give rise to concerns not only about body integrity, but can also lead to self-consciousness about how to adapt to and accept these often dramatic changes. Compared to their older counterparts, younger women are at higher risk of body image issues after breast cancer $^{11}$. From a developmental perspective, it is notable that the younger stages of life typically center on building self-confidence and developing a strong relationship with oneself and intimate others. When young breast cancer survivors are forced to adjust to significant physical changes during this sensitive developmental period, there is often a notable sense of loss around their physical functionality and attractiveness ${ }^{12}$.

Body image issues secondary to breast cancer treatment are often thought to stem from external, visible changes. These kinds of obvious body alterations include the surgical loss of breast tissues and scarring, 
chemotherapy-induced hair loss and weight change, and radiation-induced skin damage and discoloration. In addition, there may also be significant changes that are not obvious such as loss of sensation of the breast, nipple, and surrounding skin. These transformations are in turn associated with changes in women's perceptions about femininity, sensuality and with distorted, negative views of her attractiveness ${ }^{13,14}$. Subsequently, increased body image concerns in breast cancer survivors predict other domains of psychological distress, including anxiety, depression, fatigue, and a fear of cancer recurrence ${ }^{14}$.

In terms of understanding this particular negative impact of treatment for young women, permanent physical changes can act as a constant reminder of an ailing body or of a body that is vulnerable to disease. Cancer represents a breakdown of healthy boundaries, an experience when one's body feels permeable, exposed, and out of control ${ }^{8}$. Consistent with this perspective, it has been shown that heightened body image concerns are also associated with increased physical symptoms, symptoms that reflexively serve to remind women of the bodily changes that have resulted from invasive treatment ${ }^{14,15}$.

With regard to how different types of surgical intervention impact body image for young women, there is inconsistency among the findings ${ }^{16,17}$. Overall, evidence indicates that women fare better with procedures that conserve more of the breast (e.g. lumpectomy), rather than those where more tissue is removed (e.g. mastectomy). This is logical as more conservative surgeries result in less-visible changes to the breast. Similarly, studies indicate that women who undergo prophylactic mastectomies without pursuing breast reconstruction experience significantly more distress around their post-surgical appearance, feelings of femininity, and wellbeing in sexual relationships. These adverse effects were lower for many, but not all, women who chose to have reconstruction ${ }^{18,19}$. These results indicate that broadly speaking, the physical loss of the breast impacts body image, with indications that for some women, reconstruction can counter this effect. However, it is worth noting that some literature reveals no differences in well-being related to surgery type, with discussions highlighting the importance of psychological factors on how a woman experiences her surgery outcomes. The idea is that having an active role in the surgery decision can help promote feelings of empowerment and satisfaction with their body image post-surgery, regardless of the physical outcome 20,21 .

Inconsistencies in findings regarding breast reconstruction and body image likely reflect the complexity of women's perceptions of the breast. To clarify, Langellier, et al. identified four separate but closely related "breast concepts:" the "medicalized breast" represents the diseased part of the breast, the "functional breast" represents the female functionality of the breast, pertaining largely to her relationship with a child, the "gendered breast" represents femininity, physical attractiveness, and beauty, and the "sexualized breast" represents the visual and tactile experience of the breast ${ }^{22}$. As such, the decision to remove or reconstruct the breast can be conflicted, depending on how a woman identifies with various aspects of her breast. For some, there is relief from the removal of the diseased tissue. There can also be a sense of loss over the healthy, feminizing, and socially sexualized breast. For those who receive reconstruction, there can be comfort in regaining the natural appearance of the breast, but an accompanying disappointment in the sensory loss that can come with the surgical procedures used to reconstruct the breast tissue ${ }^{23}$.

Finally, it must be noted that body image is not disrupted for all young women after breast cancer. Evidence indicates that women who have a stronger body image of themselves prior to their cancer cope better with physical changes from breast cancer treatment ${ }^{10}$. This finding suggests that body image distress may be buffered by certain protective factors such as having a foundation of positive self-regard and self-confidence. Similar trends are observed in mental health distress with survivors, where lower pre-diagnostic anxiety and depression predicts lower overall psychological distress in survivorship ${ }^{5}$. Such findings have important clinical implications with regard to the importance of helping women develop compensatory coping skills in order to build a more positive foundation around body image and self-identity.

\section{SEXUAL FUNCTIONING}

Women's sexual functioning is a multifaceted experience involving physical, psychological, and interpersonal factors. For women, sexual response has been 
understood to consist of phases that rely on both psychological well-being as well as physical responsiveness. As such, to achieve a satisfying sexual experience for a woman depends as much on having a comfortable mental and interpersonal state as having a responsive and healthy physical body ${ }^{24}$. With this in mind, it is understandable that concern about risk of sexual dysfunction is particularly high in younger breast cancer survivors ${ }^{6,20}$.

For younger women, the far-reaching impact of cancer on sexual functioning is largely due to receiving a pre-menopausal breast cancer diagnosis. Chemotherapy often triggers premature ovarian failure, which in turn leads to a loss of estrogen and associated menopausal symptoms ${ }^{25}$. In addition to diminished levels of estrogen and testosterone, hormones which effect aspects of sexual functioning, menopausal changes can also include hot flashes, night flushes, mood changes, and sleep disturbances. In terms of sexual function, the marked depletion of hormones is associated with weakened libido and arousal along with changes to the vulvovaginal area, including vaginal dryness, decreased tissue elasticity, and increased tissue fragility ${ }^{26-28}$. Taken together, these physical changes cause discomfort or even pain during sexual activity, whether alone or with a partner ${ }^{29}$.

In addition to chemotherapy-induced menopause, each year tens of thousands of young breast cancer survivors also make the difficult decision to undergo ovarian suppression treatment in order to reduce their risk of cancer recurrence. Ovarian suppression can be induced by either surgically removing the ovaries or biochemically suppressing ovarian function ${ }^{30}$. These procedures similarly lead to the same severe and disruptive side effects of chemotherapy-induced menopause. Based on recently revised treatment guidelines from the American Society of Clinical Oncology, ovarian suppression is now being routinely recommended for young breast cancer survivors with a history of ER-positive breast cancer. Thus, the population of young female survivors on ovarian suppression is rapidly growing ${ }^{31}$.

Further, acute physical changes secondary to treatment-induced menopause are frequently accompanied by the subjective experience of decreased sexual interest, difficulty with sexually arousal, inability to relax and enjoy sex, and difficulty reaching orgasm ${ }^{32}$. It has been posited that for women, physical and psychological responses work in parallel to achieve sexual satisfaction ${ }^{20}$. For example, alleviating physical discomfort can often promote interest and arousal, which can then further improve the physical response. On the other hand, when a young woman finds herself feeling hopeless and/or struggling with emotional distress and negative beliefs about sexual function, such psychological and cognitive elements can in turn compound decreased sexual desire and arousal, further perpetuating sexual dysfunction ${ }^{33}$. This cycle highlights the importance of addressing both the physical and psychological impact of sexuality for survivors.

In addition, sexual dysfunction for young breast cancer survivors is also significantly associated with the quality of their intimate relationships ${ }^{32,34}$. It has been observed that survivors' perceived satisfaction in their intimate relationships is significantly correlated with sexual satisfaction, pain during sexual activity, and their overall sexual functioning, both during partnered and non-partnered activity ${ }^{32}$. Although relationships appear to be impacted by negative sexual changes, studies also indicate that women who feel more supported by their intimate partners report fewer sexual difficulties ${ }^{35}$. This again indicates the integrative and reciprocal nature of sexual function and intimacy. That is, distressing sexual function can negatively impact intimate relationships, and positive intimate relationships can confer beneficial effect on sexual function. Certainly in terms of clinical implications, this research underscores the need to potentially deliver couple-based support in order to help a couple achieve mutually satisfying intimacy, which may incorporate different sexual practices from those prior to the cancer diagnosis ${ }^{6}$.

\section{CULTURAL FACTORS}

Before offering guidance on how to concretely inquire about and address common problems regarding body image and sexual function, it is also important to acknowledge that sexuality and femininity are social constructs that vary across cultural contexts. There are historical factors and socio-cultural factors which give rise to differential trends within cultural subgroups. Of course, exceptions arise with generalizations, but being aware of trends can help inform a more culturally sensitive approach to a survivor's experience. 
Overall, evidence indicates that women across ethnicities report similar self-image concerns related to their breast cancer treatment ${ }^{36-38}$. However, studies also reveal some distinct variations, with Hispanic/ Latina women more likely than non-Hispanic/Latina women to report difficulties one year post-treatment with sexual desire, relaxing and enjoying sex, and becoming sexually aroused and having orgasms ${ }^{36}$. There are traditional and spiritual values present in the Hispanic/Latino culture around gender roles and sexuality that may be influencing these differences. For one, traditional gender roles identify the male role as more dominant, independent, and protective of the family, while the female role is traditionally characterized as being more of a caregiver, less independent, and more submissive ${ }^{39,40}$. Within this traditional perspective, virginity and female sexual purity are honored, making open communication about issues of a woman's sexuality and sexual pleasure more constrained. In keeping with this outlook, qualitative interviews have observed a trend of immigrant Hispanic/Latina breast cancer survivors feeling anxious or worried about their male partner's reactions to their missing breast and their loss of sexual desire ${ }^{41,42}$. Although Hispanic/Latina women may worry about partners' reactions to these changes, these concerns may also be buffered by good communication and perceived partner support.

Also, like most women, Hispanic/Latina women perceive cultural messages about feminine ideals and normative body image ${ }^{43}$. Traditionally, Hispanic/Latino cultures visualize the feminine ideal of a curvaceous body that includes prominent breasts. The breast in particular is associated with sensuality and fertility ${ }^{44}$. Qualitative studies of Hispanic/Latina breast cancer survivors provide clear examples of how, based on very personal negative experiences of their altered breasts post-cancer, women perceive: (i) a loss in feminine power, and (ii) a loss in their ability to attract a partner and feel attractive ${ }^{45}$. Some Hispanic/Latina breast cancer survivors have reported no longer feeling accepted as sexual partners by their husbands ${ }^{46}$. One young survivor in that study explained that based on the somewhat narrowly constrained perception of femininity in "macho" culture, she no longer feels perceived as a wife or lover by her husband, but rather only as a mother or housekeeper. This said, the traditional ideals of the Hispanic/Latina body are shifting in the contemporary culture, in particular observed in young immigrants living in the USA. Younger generations immersed in cross-cultural media are integrating Westernized body ideals, including "thinness" 43,47 ; as such, it is important to recognize that body image concerns may differ among Hispanic/Latina survivors depending on the extent to which they adopt traditional cultural views ${ }^{44}$.

Religiosity/spirituality is another cultural factor that has been studied within Hispanic/Latina breast cancer populations. Coping with the challenges of cancer through religion and spirituality has been shown to be common amongst Hispanic/Latina survivors. This trend is consistent with a cultural framework that places high value on religiosity/spirituality ${ }^{48,49}$. Some studies have found that higher levels of religiosity/ spirituality are associated with positive reports of health-related quality of life in young Hispanic/Latina breast cancer survivors ${ }^{48}$. However, this finding is inconsistent in the literature, and does not focus on quality of life issues specific to body image and sexual functioning ${ }^{50}$. The overall lack of studies on this topic calls for further research on how religious identification may be related to specific facets of body image and sexual functioning in this group.

\section{NEXT STEPS: HOW TO START}

Despite the often-severe consequences on both body image and sexual function, these issues typically go unaddressed for most young women ${ }^{51}$. From diagnosis through the survivorship phase of care, women often get little or no support around these issues and they are left feeling both isolated ${ }^{52}$ and confounded by distressing changes ${ }^{53,54}$. Given the unique and deeply distressing consequences related to body image, sexuality, and identity for young breast cancer survivors, it is imperative for clinicians to address these issues. Yet this is not an easy task. To begin, it is necessary to acknowledge a range of barriers that often prevent young patients and survivors from getting the help they need. Although Western culture is saturated with sexual content, there is a notable silence for most patients and survivors about real sexual function and problems. For the most part, clinicians do not receive formal training in speaking about sexual function with patients ${ }^{55}$ and clinicians report feeling both unprepared and embarrassed about addressing these issues ${ }^{5,56,57}$. More specifically, 
clinicians worry about upsetting or embarrassing their patients if they ask about these issues, and they also acknowledge worrying about asking questions regarding potential problems that they will not know how to answer ${ }^{58}$. There are also a myriad of practical challenges such as the lack of time and resources that clinicians cite when they are asked about barriers to communication $^{59}$.

In order to assist clinicians who want to help young women suffering from these treatment-related problems, the rest of this paper will be devoted to offering concrete guidance and helpful steps for both approaching clinical inquiry and addressing common problems.

To begin, it is essential for clinicians to have a basic framework for inquiring about these issues. Most validated sexual health assessment inventories for female cancer survivors are typically lengthy and too time-intensive to be employed for use in outpatient oncology treatment settings. However, another option to jumpstart a clinical inquiry is to use a checklist-based approach (see Table 1 in the Supplemental Material) ${ }^{60}$.

This checklist provides a straightforward roadmap for clinicians to use in a brief clinical conversation in order to identify if a woman is struggling with treatment-related sexual dysfunction. In addition, after asking about concerns on vaginal health, one might also ask if a patient has any concerns about body image. This checklist offers simple language and prompts the clinician to inquire further if necessary. For a young breast cancer survivor who is used to deafening silence in this context, the process of asking a few straightforward questions can signal an open clinical environment that validates the reality of her concerns and encourages further dialogue if needed. If a woman is satisfied with her sexual function and is not bothered by other changes, inquiry may cease and little time is wasted. However, if a young patient or survivor endorses items on this checklist, it is incumbent on the clinician to have the basic information and resources for rehabilitation available. This is especially true given that patients are less likely to raise the topic of body image concerns or sexual difficulties in clinical settings because they do not want to embarrass their doctor ${ }^{61}$. It is important to be aware that when women are accompanied by family or friends in the consulting room, they may be less comfortable answering these questions. One tip for clinicians is to announce to the patient that he or she would like to inquire about side effects of treatment, including sexual function, at which point family members or friends may choose to step out of the room during this brief part of the clinical encounter.

\section{WHAT TO DO}

Clinicians do not address these topics because they are understandably unsure of what to say if a patient endorses a problem. It is our belief that inquiry about sexual function should be rolled into a general review of systems and be approached from a similar perspective. If the message is that we inquire about sexual function no differently than about bone health, pain, and/or nausea, we de-stigmatize these issues. When patients are given appropriate education and necessary resources, they not only gain knowledge but also an increased sense of perceived competence to address the issues moving forward ${ }^{62}$. However, it is understandable that a clinician might avoid asking if he or she is not sure how to proceed with an endorsement of a problem.

\section{Body image}

If a woman endorses concern or distress about changes in body image, it is important to convey the essential idea that adjustment is a process that depends upon both acceptance and change. The process of improving body image involves identifying the changes one needs to adapt to (e.g. loss of nipple sensation, alterations in physical appearance), and actively making changes that lead to improved physical strength, body integrity, and self-efficacy. A handful of studies have reported on interventions aimed at addressing difficulties with body image in survivors. One useful approach is using an exercise-based intervention. Of two previous studies, one assigned breast cancer survivors twice-weekly resistance training for two months ${ }^{63}$, and the second provided twice-weekly strength training in a group guided by a trained fitness professional for 13 weeks, followed by encouragement to continue exercises independently for one year ${ }^{64}$. In each study, both younger and older survivors reported significant improvements in body image concerns up to one year after completing the 
intervention. Both studies imply the importance of exercise in improving women's self-confidence and control over their bodies, in addition to building physical strength. Thus, clinicians can suggest that starting a regular, structured exercise program can provide the combined benefit of improving post-treatment physical capacity and strength while also improving women's satisfaction with physical appearance. Both factors buffer the negative impact cancer treatments can have on women's perceived body "wholeness" and attractiveness.

A secondary approach to helping women improve body image rests on using a counseling approach, either individually or as a couple. One previous study provided six sessions of couples and sex therapy with women and their partners starting two days prior to mastectomy surgery. Sessions involved a combination of communication training, exposing the mastectomy scars to the couple, sensate focus, and body imagery ${ }^{65}$. Significant improvements were observed in women's body image both when looking at themselves and when facing their partner, as well as improvements in certain domains of sexual functioning. Similarly, other ongoing studies using couples-based interventions for women after breast cancer show promising results ${ }^{66}$. Thus, if a woman notes that body image is of concern and conveys worry about the impact of breast cancer on her relationship, couples therapy may be appropriate. However, being sensitive to cultural differences, some women may not feel at ease discussing such issues with a partner and would be better served to work with a counselor individually.

\section{Sexual health}

In terms of addressing sexual dysfunction, as has been previously suggested, the majority of young breast cancer patients often need a limited number of simple solutions to address common problems such as vaginal dryness, pain with intercourse, and decreased arousal ${ }^{67}$. Women need to be informed about the basics of addressing vaginal health after breast cancer treatment, including the need to restore moisture, stretch, and blood flow to vaginal tissue. Women should be counseled about the use of vaginal moisturizers and lubricants as well as pelvic floor exercises that can directly help improve sexual function. For example, a woman and/or her partner may simply not know that lack of vaginal moisture after breast cancer is directly related to loss of natural estrogen, and that this situation is not a reflection of a woman's ability to become sexually aroused or her capacity to be sexually satisfied. However, when a woman or her partner has this misconception, this can lead to consequences such as emotional distancing, loss of sexual self-esteem, and confirmation of a woman's negative conception that she is no longer feminine or capable of being a fulfilling sexual partner. A 2011 paper by Carter, et al. overviews a detailed presentation of vaginal health promotion after cancer ${ }^{67}$, and a more recent paper by Coady, et al. ${ }^{68}$ offers a similarly detailed overview of how to address treatment-related sexual pain. Table 2 in the Supplemental Material provides an example of a handout that has been used with Spanish-speaking patients and can be given directly to women. This "tip sheet" provides an overview of many of the basic strategies that young women need to be aware of regarding sexual health.

In summary, we encourage clinicians to be sensitive to both the physical and psychological aspects of the equation. Because the issues of body image and sexual function are truly at the nexus of psychological, physical, and interpersonal factors, it is helpful for clinicians to identify individual counselors, social workers, and/or couples therapists that they can potentially refer to. In general, it is our strong recommendation that inquiry is made about body image and sexual health in parallel with any other review of systems. Comparable to the way that women may be asked about pain, nausea, or fatigue, the checklist that we have provided offers a straightforward model for how to ask about these common, distressing problems that are often completely ignored. Inquiry is an important initial step as it opens the door to providing women who endorse difficulties with effective interventions. Although intervention research in sexual health is gaining attention, further study is needed around developing and implementing treatment across groups. In particular, there is an enormous unmet need to evaluate the effectiveness of strategies with low to middle income populations and with women receiving community-based care. Research is also needed to translate and adapt current interventions across cultural contexts.

Given the enormous emphasis that understandably goes to saving lives of young women with breast cancer, it is critical we pay attention to the quality of life 
after treatment ends. As positive body image and satisfying sexual function are life-affirming and restorative by nature, we are undoubtedly responsible for doing everything we can to help women repair these experiences and live fully long after their diagnosis of breast cancer.

\section{SUPPLEMENTARY DATA}

Supplementary data is available at Revista de Investigación Clínica online (www.clinicalandtranslationalinvestigation.com). These data are provided by the corresponding author and published online for the benefit of the reader. The contents of supplementary data are the sole responsibility of the authors.

\section{REFERENCES}

1. Bleyer A, Barr R, Hayes-Lattin B, Thomas D, Ellis C, Anderson B. The distinctive biology of cancer in adolescents and young adults. Nat Rev Cancer. 2008;8:288-98.

2. Howlader N, Noone AM, Krapcho M, et al. SEER Cancer Statistics Review, 1975-2013. National Cancer Institute. 2016.

3. Anderson WF, Chatterjee N, Ershler WB, Brawley OW. Estrogen receptor breast cancer phenotypes in the Surveillance, Epidemiology, and End Results database. Breast Cancer Res Treat. 2002; 76:27-36

4. Anders CK, Fan C, Parker JS, et al. Breast carcinomas arising at a young age: unique biology or a surrogate for aggressive intrinsic subtypes? J Clin Oncol. 2011:29:e18-20.

5. Fobair P, Stewart SL, Chang S, D'Onofrio C, Banks PJ, Bloom JR. Body image and sexual problems in young women with breast cancer. Psychooncology. 2006;15:579-94.

6. Bober SL, Varela VS. Sexuality in adult cancer survivors: challenges and intervention. J Clin Oncol. 2012;30:3712-9.

7. Kasper AS. A feminist, qualitative methodology: a study of women with breast cancer. Qualitative Sociology. 1994;17:263-81.

8. Waskul DD, van der Riet P. The abject embodiment of cancer patients: dignity, selfhood, and the grotesque body. Symbolic Interaction. 2002;25:487-513.

9. Cohen MZ, Kahn DL, Steeves RH. Beyond body image: the experience of breast cancer. Oncol Nurs Forum. 1998;25:835-41.

10. Han J, Grothuesmann D, Neises M, Hille U, Hillemanns P. Quality of life and satisfaction after breast cancer operation. Arch Gynecol Obstet. 2010;282:75-82

11. Bakht S, Najafi S. Body image and sexual dysfunctions: comparison between breast cancer patients and healthy women. Procedia-Social Behav Sci. 2010;5:1493-7.

12. Paterson CL, Lengacher CA, Donovan KA, Kip KE, Tofthagen CS. Body image in younger breast cancer survivors: a systematic review. Cancer Nurs. 2016;39:E39-58.

13. Liu J, Peh CX, Mahendran R. Body image and emotional distress in newly diagnosed cancer patients: the mediating role of dysfunctional attitudes and rumination. Body Image. 2016; 20:58-64

14. Przezdziecki A, Sherman KA, Baillie A, Taylor A, Foley E, StalgisBilinski K. My changed body: breast cancer, body image, distress and self-compassion. Psychooncology. 2013;22:1872-9.

15. Miller SJ, Schnur JB, Weinberger-Litman SL, Montgomery GH The relationship between body image, age, and distress in women facing breast cancer surgery. Palliat Support Care. 2014; 12:363-7

16. Yurek D, Farrar W, Andersen BL. Breast cancer surgery: comparing surgical groups and determining individual differences in postoperative sexuality and body change stress. J Consult Clin Psychol. 2000;68:697-709.
17. Engel J, Kerr J, Schlesinger-Raab A, Sauer H, Holzel D. Quality of life following breast-conserving therapy or mastectomy: results of a 5-year prospective study. Breast J. 2004;10:223-31.

18. Anderson C, Islam JY, Elizabeth Hodgson M, et al. Long-term satisfaction and body image after contralateral prophylactic mastectomy. Ann Surg Oncol. 2017;29:e18-20.

19. Frost MH Schaid DJ, Sellers TA, et al. Long-term satisfaction and psychological and social function following bilateral prophylactic mastectomy. JAMA. 2000;284:319-24.

20. Pinto AC. Sexuality and breast cancer: prime time for young patients. J Thorac Dis. 2013:5(Suppl 1):S81-6.

21. Kedde H, van de Wiel HB, Weijmar Schultz WC, Wijsen C. Sexual dysfunction in young women with breast cancer. Support Care Cancer. 2013:21:271-80.

22. Langellier KM, Sullivan CF. Breast talk in breast cancer narratives. Qual Health Res. 1998;8:76-94

23. Kwait RM, Pesek S, Onstad M, et al. Influential forces in breast cancer surgical decision making and the impact on body image and sexual function. Ann Surg Oncol. 2016;23:3403-11.

24. Wylie K, Mimoun S. Sexual response models in women. Maturitas. 2009;63:112-5.

25. Anchan RM, Ginsburg ES. Fertility concerns and preservation in younger women with breast cancer. Crit Rev Oncol Hematol. 2010;74:175-92.

26. Dennerstein L, Dudley EC, Hopper JL, Burger H. Sexuality, hormones and the menopausal transition. Maturitas. 1997;26:83-93.

27. Krebs LU. Sexual health during cancer treatment. In: Quinn GP, Vadaparampil ST, editors. Reproductive Health and Cancer in Adolescents and Young Adults: Springer Netherlands; 2012. p. 61-76.

28. Simon J, Braunstein G, Nachtigall L, et al. Testosterone patch increases sexual activity and desire in surgically menopausal women with hypoactive sexual desire disorder. J Clin Endocrinol Metab. 2005;90:5226-33.

29. Falk SJ, Bober S. Vaginal Health During Breast Cancer Treatment. Curr Oncol Rep. 2016;18:1-5.

30. Graziottin A, Basson R. Sexual dysfunction in women with premature menopause. Menopause. 2004;11:766-77.

31. Burstein $\mathrm{HJ}$, Lacchetti $\mathrm{C}$, Anderson $\mathrm{H}$, et al. Adjuvant endocrine therapy for women with hormone receptor-positive breast cancer: American society of clinical oncology clinical practice guideline update. J Oncol Pract. 2015;12:390-3.

32. Boquiren VM, Esplen MJ, Wong J, Toner B, Warner E, Malik N. Sexual functioning in breast cancer survivors experiencing body image disturbance. Psychooncology. 2016;25:66-76.

33. Barlow DH. Causes of sexual dysfunction: the role of anxiety and cognitive interference. J Consult Clin Psychol. 1986;54:140-8.

34. Schover LR, van der Kaaij M, van Dorst E, Creutzberg C, Huyghe E, Kiserud CE. Sexual dysfunction and infertility as late effects of cancer treatment. EJC Suppl. 2014;12:41-53.

35. Kinsinger SW, Laurenceau JP, Carver CS, Antoni MH. Perceived partner support and psychosexual adjustment to breast cancer. Psychol Health. 2011:26:1571-88.

36. Christie KM, Meyerowitz BE, Maly RC. Depression and sexual adjustment following breast cancer in low-income Hispanic and non-Hispanic White women. Psychooncology. 2010;19. 1069-77

37. Giedzinska AS, Meyerowitz BE, Ganz PA, Rowland JH. Healthrelated quality of life in a multiethnic sample of breast cancer survivors. Ann Behav Med. 2004:28:39-51.

38. Fobair P, Stewart SL, Chang S, D'Onofrio C, Banks PJ, Bloom JR. Body image and sexual problems in young women with breast cancer. Psychooncology. 2006;15:579-94.

39. Gloria AM, Ruiz EL, Castillo EM. Counseling and psychotherapy with Latino and Latina clients. In: Smith TB, editor. Practicing multiculturalism: affirming diversity in counseling and psychology. Boston: Pearson Education; 2004. p. 167-89.

40. Espin OM. Cultural and historical influences on sexuality in Hispanic/Latin women: implications for psychotherapy. In: Vance CA, editor. Pleasure and danger: exploring female sexuality. Boston: Routledge \& Kegan Paul; 1984. p. 149-63.

41. Martinez-Ramos GP, Biggs MJG, Lozano Y. Quality of life of Latina breast cancer survivors: from silence to empowerment. Advances Social Work. 2013;14:82-101.

42. Lopez-Class M, Perret-Gentil M, Kreling B, Caicedo L, Mandelblatt J, Graves KD. Quality of life among immigrant Latina breast cancer survivors: realities of culture and enhancing cancer care. J Cancer Educ. 2011;26:724-33.

43. Viladrich A, Yeh MC, Bruning N, Weiss R. "Do Real Women Have Curves?": paradoxical body images among Latinas in New York City. J Immigr Minor Health. 2009;11:20-8. 
44. Gil RM, Vazquez $\mathrm{Cl}$. The Maria paradox: how Latinas can merge old world traditions with new world self-esteem. New York: Open Road Media; 2014.

45. Buki LP, Reich M, Lehardy EN. "Our organs have a purpose": body image acceptance in Latina breast cancer survivors. Psychooncology. 2016;25:1337-42.

46. Ashing-Giwa KT, Padilla GV, Bohorquez DE, Tejero JS, Garcia M. Understanding the breast cancer experience of Latina women. J Psychosoc Oncol. 2006;24:19-52.

47. Pompper D, Koenig J. Cross-cultural-generational perceptions of ideal body image: Hispanic women and magazine standards. Journalism Mass Communication Quarterly. 2004;81:89-107.

48. Wildes KA, Miller AR, de Majors SS, Ramirez AG. The religiosity/ spirituality of Latina breast cancer survivors and influence on health-related quality of life. Psychooncology. 2009;18:831-40.

49. Culver JL, Arena PL, Antoni MH, Carver CS. Coping and distress among women under treatment for early stage breast cancer: comparing African Americans, Hispanics and non-Hispanic Whites. Psychooncology. 2002;11:495-504.

50. Yanez B, Thompson EH, Stanton AL. Quality of life among Latina breast cancer patients: a systematic review of the literature. J Cancer Surviv. 2011;5:191-207.

51. Katz A. The sounds of silence: sexuality information for cancer patients. J Clin Oncol. 2005;23:238-41.

52. Gilbert E, Ussher JM, Perz J, Wong WK, Hobbs K, Mason C. Men's experiences of sexuality after cancer: a material discursive intra-psychic approach. Cult Health Sex. 2013;15:881-95.

53. Flynn KE, Reese JB, Jeffery DD, et al. Patient experiences with communication about sex during and after treatment for cancer. Psychooncology. 2012;21:594-601.

54. Park ER, Norris RL, Bober SL. Sexual health communication during cancer care: barriers and recommendations. Cancer J. 2009. 15:74-7.

55. Bober SL, Recklitis CJ, Campbell EG, et al. Caring for cancer survivors: a survey of primary care physicians. Cancer. 2009 ; 115:4409-18.

56. Hordern AJ, Street AF. Constructions of sexuality and intimacy after cancer: patient and health professional perspectives. Soc Sci Med. 2007;64:1704-18.
57. Stead ML, Brown JM, Fallowfield L, Selby P. Lack of communication between healthcare professionals and women with ovarian cancer about sexual issues. Br J Cancer. 2003;88:666-71.

58. Isidori AM, Pozza C, Esposito K, et al. Development and validation of a 6 -item version of the female sexual function index (FSFI) as a diagnostic tool for female sexual dysfunction. J Sex Med. 2010;7:1139-46.

59. Wiggins DL, Wood R, Granai CO, Dizon DS. Sex, intimacy, and the gynecologic oncologists: survey results of the New England Association of Gynecologic Oncologists (NEAGO). J Psychosoc Oncol. 2007;25:61-70.

60. Bober S, Reese JB, Barbera L, et al. How to ask and what to do: a guide for clinical inquiry and intervention regarding female sexual health after cancer. Curr Opin Support Palliat Care. 2016:44-54.

61. Hordern AJ, Street AF. Let's talk about sex: risky business for cancer and palliative care clinicians. Contemp Nurse. 2007;27: 49-60.

62. Deci EL, Ryan RM. Self-determination theory in health care and its relations to motivational interviewing: a few comments. Int J Behav Nutr Phys Act. 2012;9:24.

63. Benton MJ, Schlairet MC, Gibson DR. Change in quality of life among breast cancer survivors after resistance training: is there an effect of age? J Aging Phys Act. 2014;22:178-85.

64. Speck RM, Gross CR, Hormes JM, et al. Changes in the Body Image and Relationship Scale following a one-year strength training trial for breast cancer survivors with or at risk for lymphedema. Breast Cancer Res Treat. 2010;121:421-30.

65. Kalaitzi C, Papadopoulos VP, Michas K, Vlasis K, Skandalakis P, Filippou D. Combined brief psychosexual intervention after mastectomy: effects on sexuality, body image, and psychological well-being. J Surg Oncol. 2007;96:235-40.

66. Reese JB, Porter LS, Casale KE, et al. Adapting a couple-based intimacy enhancement intervention to breast cancer: a developmental study. Health Psychol. 2016;35:1085-96.

67. Carter J, Goldfrank D, Schover LR. Simple strategies for vaginal health promotion in cancer survivors. J Sex Med. 2011; 8:549-59.

68. Coady D, Kennedy V. Sexual health in women affected by cancer: focus on sexual pain. Obstet Gynecol. 2016;128:775-91. 